\title{
Video Article \\ Indoor Experimental Assessment of the Efficiency and Irradiance Spot of the Achromatic Doublet on Glass (ADG) Fresnel Lens for Concentrating Photovoltaics
}

\author{
Guido Vallerotto ${ }^{1}$, Marta Victoria ${ }^{1}$, Stephen Askins ${ }^{1}$, Ignacio Antón ${ }^{1}$, Gabriel Sala ${ }^{1}$, Rebeca Herrero ${ }^{1}$, César Domínguez \\ ${ }^{1}$ Instituto de Energía Solar, Universidad Politécnica de Madrid
}

Correspondence to: Guido Vallerotto at guido.vallerotto@ies-def.upm.es

URL: https://www.jove.com/video/56269

DOI: doi:10.3791/56269

Keywords: Engineering, Issue 128, Solar energy, concentrators, aberration compensation, Fresnel lens, optical design, optical characterization

Date Published: 10/27/2017

Citation: Vallerotto, G., Victoria, M., Askins, S., Antón, I., Sala, G., Herrero, R., Domínguez, C. Indoor Experimental Assessment of the Efficiency and Irradiance Spot of the Achromatic Doublet on Glass (ADG) Fresnel Lens for Concentrating Photovoltaics. J. Vis. Exp. (128), e56269, doi:10.3791/56269 (2017).

\section{Abstract}

We present a method to characterize achromatic Fresnel lenses for photovoltaic applications. The achromatic doublet on glass (ADG) Fresnel lens is composed of two materials, a plastic and an elastomer, whose dispersion characteristics (refractive index variation with wavelength) are different. We first designed the lens geometry and then used ray-tracing simulation, based on the Monte Carlo method, to analyze its performance from the point of view of both optical efficiency and the maximum attainable concentration. Afterwards, ADG Fresnel lens prototypes were manufactured using a simple and reliable method. It consists of a prior injection of plastic parts and a consecutive lamination, together with the elastomer and a glass substrate to fabricate the parquet of ADG Fresnel lenses. The accuracy of the manufactured lens profile is examined using an optical microscope while its optical performance is evaluated using a solar simulator for concentrator photovoltaic systems. The simulator is composed of a xenon flash lamp whose emitted light is reflected by a parabolic mirror. The collimated light has a spectral distribution and an angular aperture similar to the real Sun. We were able to assess the optical performance of the ADG Fresnel lenses by taking photographs of the irradiance spot cast by the lens using a charge-coupled device (CCD) camera and measuring the photocurrent generated by several types of multi junction (MJ) solar cells, which have been previously characterized at a solar simulator for concentrator solar cells. These measurements have demonstrated the achromatic behavior of ADG Fresnel lenses and, as a consequence, the suitability of the modelling and manufacturing methods.

\section{Video Link}

The video component of this article can be found at https://www.jove.com/video/56269/

Introduction

Concentrator photovoltaic (CPV) is a promising technology to reduce the cost of solar-based electricity because this technology can take advantage of the rapid incremental improvement in the efficiency of advanced multi junction (MJ) solar cells. These devices are composed of several sub-cells (usually three named as top, middle, and bottom) each of which is made of a different semiconductor compound. Every subcell has a different bandgap resulting in a different spectral response, which enables each to convert a distinct part of the solar spectrum into electricity. In this way, MJ solar cells are capable of exploiting a wide range of the solar spectrum (typically $300-1800 \mathrm{~nm}$ ) achieving efficiency values higher than $46 \%$ under concentrated light ${ }^{1}$. In order to compensate for the high cost of such photovoltaic devices, optical systems are used to concentrate the irradiance on them, which reduces the final system cost. Currently, most of the commercially available high concentration photovoltaic (HCPV) systems are based on silicone-on-glass (SoG) hybrid Fresnel lenses ${ }^{2}$. In all refractive optical systems, chromatic aberration is the factor most severely decreasing the lens performance in terms of the maximum attainable concentration ${ }^{3}$ (i.e., minimum light spot area). Making use of an achromatic lens, that is, a lens with highly reduced chromatic aberration, it is possible to significantly increase the maximum attainable concentration without a need for any additional optical elements (referred to as secondary optical elements ${ }^{4,5}$ ).

The design of achromatic lenses (commonly called achromatic doublets because they are fabricated coupling two materials with different dispersion characteristics) has been well known since the 18th century. The conventional achromatic doublet is composed of two different glasses: the first one is called the crown and has low dispersion, while the second one is called the flint and has high dispersion. However, the overall cost of these kinds of glasses and their processing makes them unaffordable for HCPV systems. Languy and co-authors proposed an achromatic doublet for CPV composed of two plastics: poly (methyl methacrylate) (PMMA) and polycarbonate (PC) ${ }^{6}$. In their article, a comparative analysis on the different configurations and their advantages is presented but without addressing their manufacturability and scalability at high production.

The ADG Fresnel lens proposed here has been designed in such a way that light at a certain short wavelength ("blue" light) and a certain long wavelength ("red" light) have exactly the same focal distance. Details of the design method for standard achromatic doublets can be found elsewhere ${ }^{7}$. Several ray-tracing simulations have been carried out to demonstrate the improvements obtained using an ADG Fresnel lens 
instead of a conventional SoG Fresnel lens. A detailed report on the obtained results was presented $\mathrm{in}^{4}$. The most important result is that when substituting a conventional SoG Fresnel lens with an ADG Fresnel lens, the attainable concentration increases about three times while maintaining the same optical efficiency. Furthermore, since the manufacturing process ${ }^{8}$ envisaged to obtain the ADG is very similar to the one employed to fabricate SoG lenses, the increase in concentration will be obtained without significantly increasing the cost.

Here we present a protocol to perform a comprehensive characterization of concentrators comprising a refractive primary lens and we apply this protocol to both a conventional SoG Fresnel lens (used as a benchmark) and several ADG Fresnel lens prototypes. To do so, a solar simulator for CPV has been used. A detailed description of the simulator and all its components, as well as its operating principles, has been presented elsewhere ${ }^{9}$.

\section{Protocol}

\section{Lens Modelling using Ray-Tracing Simulation}

1. Model preparation

1. Import ADG Fresnel lens geometry into ray-tracing simulation software and set up material properties such as transmittance and refractive index.

NOTE: The ADG Fresnel design has been developed at the Solar Energy Institute and it consists of computer code based on basic optics principles such as Fermat's principle and Snell's law. Dispersion curves of the materials composing the lens have been used to develop the design method. A detailed description of the design method is presented elsewhere ${ }^{4}$.

2. Set up a light source with real properties of the sun such as angular aperture and spectral distribution.

3. Place a receiver at a distance from the lens equal to the nominal focal distance.

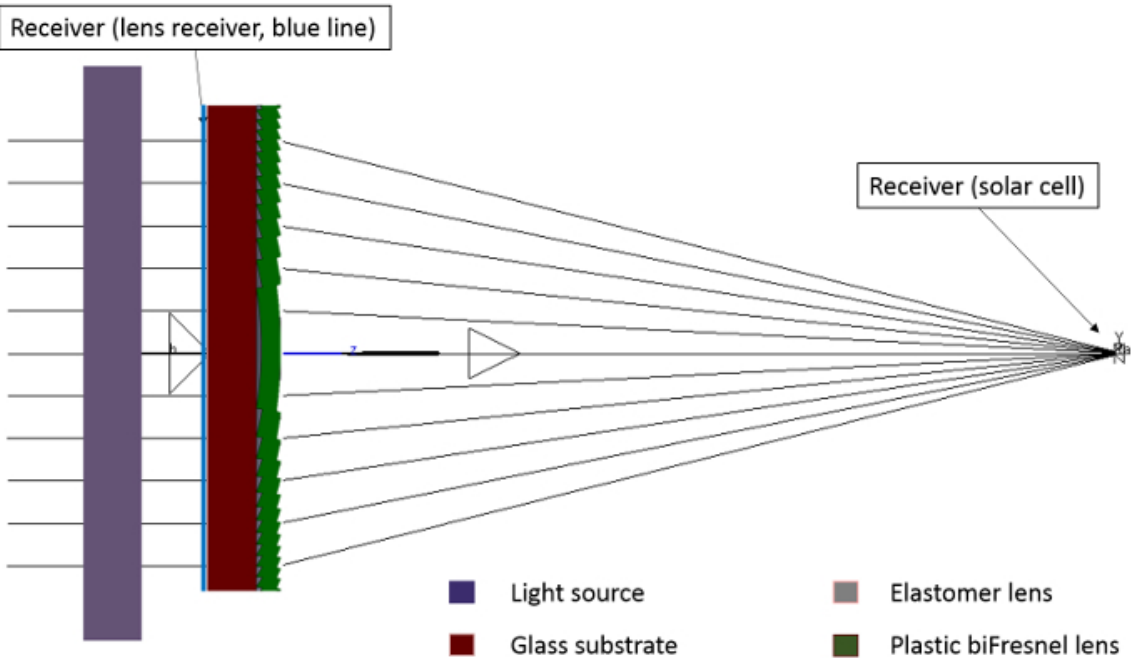

Figure 1. Screenshot of the ray-tracing simulation model. It is possible to observe the light source, the ADG Fresnel lens (comprising the glass substrate, the elastomer, and the plastic bi-Fresnel lens), and the receivers used to measure the irradiance at the lens aperture (lens receiver) and irradiance at the exit (solar cell receiver). Please click here to view a larger version of this figure.

2. Run the simulation and calculate desired results such as the maximum attainable concentration and lens optical efficiency. Attainable concentration is defined as the ratio between the lens optical aperture and the area of the receiver where the spot is cast. Optical efficiency is defined as the ratio between the power at the receiver and the power at the lens optical aperture ${ }^{10}$.

NOTE: The area of the receiver is much larger than the light spot cast by the lens in order to ensure that the receiver collects every ray transmitted by the lens. In this way, the calculated optical efficiency takes into account losses due to materials absorption, reflection, and manufacturing constraints (draft angles and tip rounding at corner and valleys).

3. Repeat steps 1.1. and 1.2. simulating a conventional silicone-on-glass (SoG) Fresnel instead of an ADG Fresnel lens to be used as benchmark. 


\section{Solar Cell Characterization}

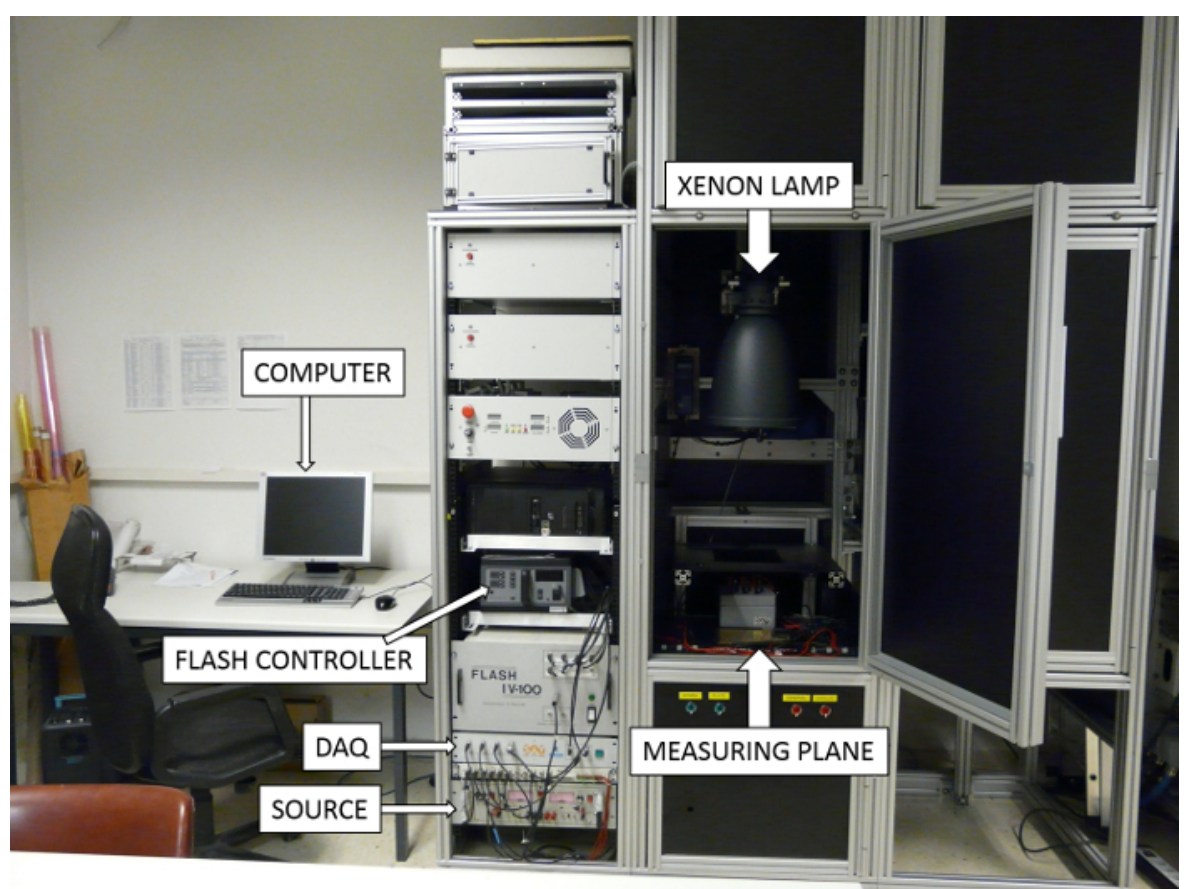

Figure 2. Solar simulator for concentrator solar cells. Photograph of the solar simulator used to characterize solar cells under concentrated irradiance. On the top of the figure, it is possible to observe the lamp whose position determines the concentration level. On the bottom, the measuring plane with reference component solar cells and the DUT is shown. On the left of the photograph, it is possible to appreciate the electronic equipment (power supply and DAQ) and the computer used to perform the characterization. Please click here to view a larger version of this figure.

1. Calibration of solar simulator for solar cell characterization

1. Place inside the solar simulator the reference component cells (top, middle, and bottom), also known as isotypes, which were calibrated under a reference spectrum and the device under test (DUT), that is, the solar cell to be measured.

NOTE: Place the reference cells and DUT as close together as possible in order to reduce possible errors due to non-uniform illumination at the measuring plane.

2. Adjust the flash lamp positioning (height) in order to reach the desired level of concentration. The further the lamp is from the measuring plane, the lower the concentration achieved.

1. The spectral distribution depends on the position of the lamp and the flash intensity. Add the necessary filters to adjust the spectral distribution. The procedure to obtain a distribution similar to the reference spectrum is described in step 2.2.1.

3. Connect the isotypes and DUT to the Data Acquisition (DAQ) board of the solar simulator.

4. Using a text editor, create a text file containing the polarization values to be used in the cell current-voltage (IV) curve measurement. The text file contains one line per voltage point. More voltage points result in higher curve definition. Since all the involved solar cells are $\mathrm{MJ}$ solar cells, the polarization values are comprised of values between $0 \mathrm{~V}$ and $3.1 \mathrm{~V}$.

\section{Measurements}

1. The light intensity throughout the flash decay has an initial peak and then starts to decrease (Figure 3). The light spectral distribution is also modified throughout the flash pulse. A conventional MJ solar cell is composed of three sub-cells with different bandgaps that are connected in series. Each sub-cell can convert electricity in a different part of the solar spectrum. Therefore, the current generated by the MJ solar cell is always limited by the sub-cell producing the least current. To perform an accurate measurement, select an irradiance level where both isotypes, corresponding to the top and middle sub-cells, indicate exactly the same irradiance level. This confirms that the cell is measured under the target concentration level and spectrum. The fact that the irradiance level indicated by the bottom sub-cell is not coincident can be neglected. This is because commercial Ge-based MJ solar cells are never current limited by this sub-cell. Figure 3 depicts a graphical explanation of this procedure.

2. Once the desired irradiance level for measurement is identified, start the IV test. The simulator reads polarization points from the text file defined in step 2.1.4.; for every point, the equipment polarizes the cell at the desired voltage, triggers the flash, and measures the current generated by the solar cell. The pair of current and voltage values, that is the IV curve, is displayed on the computer screen. NOTE: From the IV curve, it is possible to obtain the short-circuit current $\left(\mathrm{I}_{\mathrm{sc}}\right)$, open circuit voltage $\left(\mathrm{V}_{\mathrm{oc}}\right)$, fill factor $(\mathrm{FF})$, and efficiency of the DUT (even though in the next sections, only the short circuit current is used).

3. Repeat step 2.2.2. at different concentration levels to check that the solar cell photocurrent depends linearly on the concentration level (see Figure 4) and, hence, the calibrated cell can be used as a light sensor to determine the irradiance at the lens focal plane. For each concentration level, adjust the spectral distribution of the flash light using appropriate filters in order to perform measurements when both isotypes, top and middle sub-cells, indicate the same irradiance level, as explained in step 2.2.1. 


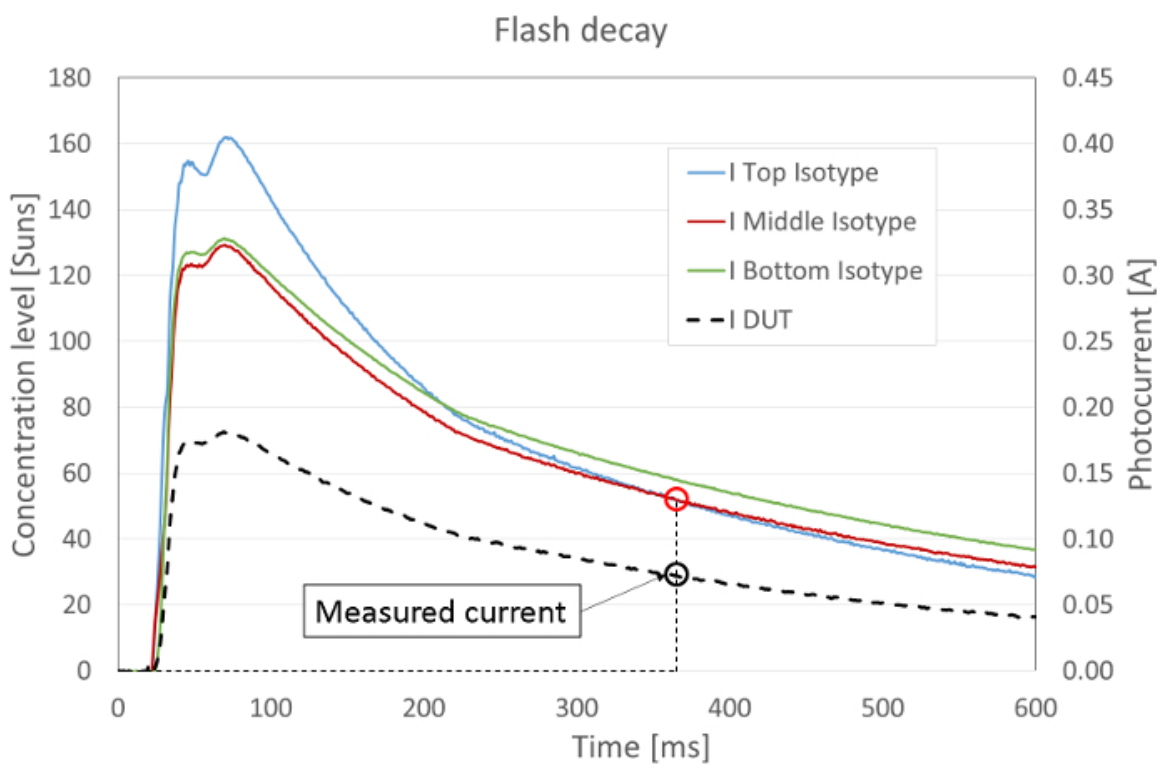

Figure 3. Time evolution of the measured magnitudes throughout the flash decay. On the graph, it has been marked the instant when the isotype cells, corresponding to the top and middle sub-cells, measure the same irradiance level. Following the black dashed line which starts from the intersection of the curves corresponding to top and middle subcells, it is possible to identify the DUT current value (black circle) as the current measured in the precise moment in which top and middle reference sub-cells see the same irradiance level. Please click here to view a larger version of this figure.

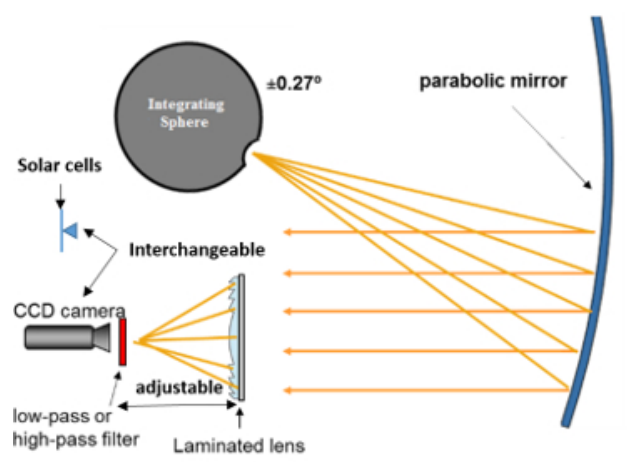

(a)

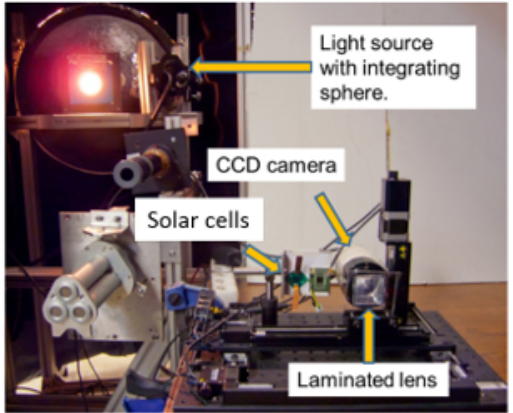

(b)

Figure 4. (A) Scheme of the setup used to carry out experimental testing. (B) Photograph of the experimental setup and its components (light source with integrating sphere, lens sample, CCD camera, and solar cells used as light sensors). The parabolic mirror and filters are not visible in this photograph. Please click here to view a larger version of this figure. 


\section{Lens Characterization.}

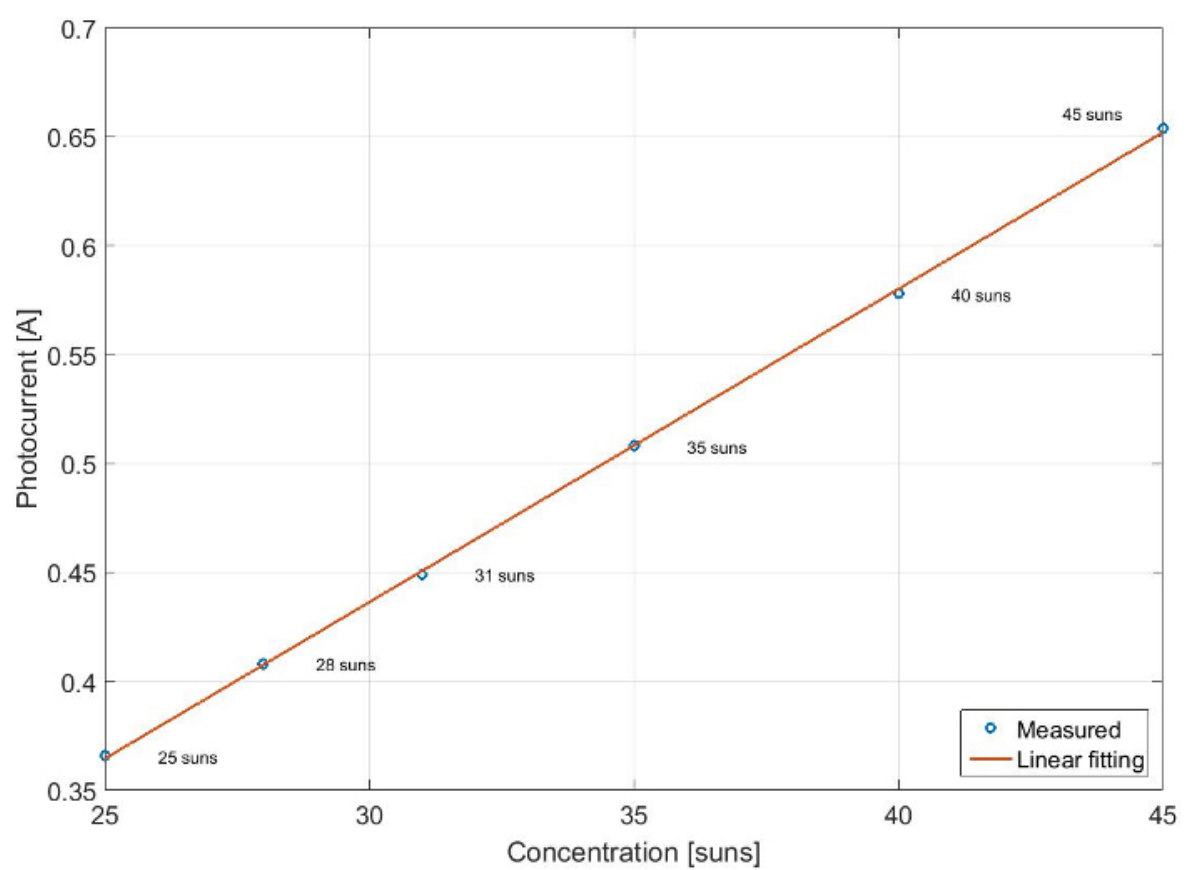

Figure 5. Graph representing the evolution of the photocurrent generated by a MJ solar cell as a function of the concentration. As expected there is a linear dependency. Please click here to view a larger version of this figure.

1. Setup preparation.

1. Mount the 3-axes automated positioning platform: a computer assisted moving platform able to accurately control the relative position between the calibrated solar cell/CCD camera and the lens to be measured.

1. Check that the 3-axes automated positioning platform is perfectly horizontal using a bubble level.

2. Mount the solar cell/CCD camera support on the platform's moving holder in such a way that it is possible to control its position along $x$, $y$ and $z$ axes.

3. Mount the lens support in the platform in front of the moving holder described in step 3.1.2. Using the moving holder on the $x$ and $y$ axes, it is possible to perfectly center the lens with respect to the solar cell/CCD camera objective. Moving the holder along the $z$ axis, it is possible to place the solar cell/CCD camera objective in the optimal focal point of the lens (minimum spot size) and to move it along its optical axis.

4. Connect every device (automated positioning platform, DAQ board to measure the cell photocurrent, CCD camera, and xenon lamp) to the computer used to perform the entire experimental test.

5. Test the connection and operation of all connected devices.

1. Open the software controlling the solar simulator for CPV and press the button "light pulse" in order to shoot a flash. If the flash decay graph looks similar to Figure 3, it means that DAQ board, xenon lamp, isotype subcells, and DUT are working properly.

2. Open the software controlling the CCD camera to check that the camera is working properly.

3. Open the software controlling the computer assisted moving platform and use it to move the moving holder along the three axes. To do so, select one axis among the axes listed on the top left of the software window, then insert a position in "move absolute" and pulse "run". If the moving holder moves as expected, it means that the moving platform is working properly.

6. Clean and place the lens to be measured on the fixed support mounted on the automated positioning platform.

7. In front of the sensor, place either a hot mirror (short-pass filter blocking light whose wavelength is longer than $700 \mathrm{~nm}$ ) or a cold mirror (long pass filter blocking light whose wavelength is shorter than $700 \mathrm{~nm}$ ).

NOTE: Step 3.1.7. is necessary only for measurements using the CCD camera.

8. Use the moving holder to center the solar cell/CCD camera with respect to the lens and place it at the optimal focal point.

9. Using any text editor, create a text file containing in every line the coordinates corresponding to a measurement point (a certain lens-toreceiver distance) starting from a position of the cell/CCD camera $5 \mathrm{~mm}$ closer to the lens than the optimal focal distance and up to a position $5 \mathrm{~mm}$ further.

\section{Measurement phase}

1. Solar cell measurements

NOTE: In the same way as the solar simulator for solar cells described in the previous section, the light intensity and the spectral distribution of the solar simulator for CPV changes throughout the flash decay. The graphic representation of the flash decay is similar to the one obtained with the solar simulator for concentrator cells described in step 2.2.1. and depicted in Figure 3. There is an initial peak and then it decreases. The light spectral distribution changes throughout the flash decay. The measurement is performed at the moment where both isotypes, corresponding to top and middle sub-cells, indicate the same irradiance level.

NOTE: Contrary to the case of the solar simulator for solar cells in this case the only control we have over the irradiance level is the flash light intensity and neutral filters 
1. Once the optimal irradiance level has been identified, it is possible to begin the test. For every position defined in step 3.1.9., trigger the flash light. The simulator then generates a text file containing data signals throughout the flash decay from which it is possible to deduce solar cell current generation under light concentrated by the lens.

2. Repeat steps from 3.1.7. to 3.2.1.3. for every lens to be measured.

\section{CCD camera measurements}

1. For every position defined in 3.1.9., using the CCD camera, take a photograph of the generated light spot. NOTE: The CCD sensor of the camera coupled with a hot or cold mirror has a spectral response similar to the top and middle sub-cell, respectively (see Figure 6). Furthermore, in order to get photographs with useful information, it is necessary to take some precautions. Firstly, the light intensity of the flash must be adjusted in order to get a good signal-to-noise ratio and, at the same time, not saturate the CCD sensor. To do so, it is possible to directly modify the flash intensity or to use neutral filters to get the desired irradiance level. Secondly, it is important that the simulator chamber is completely dark to avoid the influence of external light sources on measurements.

2. Temperature measurements

1. Place the lens to be measured inside the thermal chamber used to control the lens temperature during the test.

2. Using the thermal chamber, vary the lens temperature from $10{ }^{\circ} \mathrm{C}$ to $50^{\circ} \mathrm{C}$ with steps equal to $10^{\circ} \mathrm{C}$. To do so, place the lenses inside a thermal chamber with a transparent front cover.

3. Carry out measurement for different temperatures using the CCD camera in the same way described in 3.2.2.1. NOTE: The temperature of the lens being tested is directly measured through thermocouples attached to it. The temperature difference across the lenses surface is lower than $2{ }^{\circ} \mathrm{C}$.

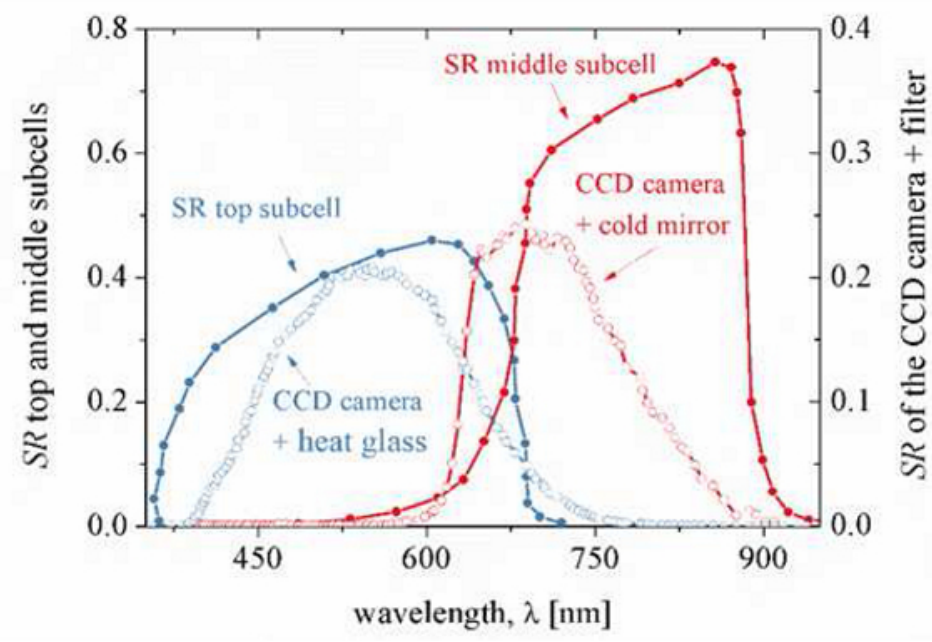

Figure 6. Spectral response (SR) of the CCD camera silicon sensor filtered by a cold mirror or a heat glass (empty dots) to simulate the SR of the middle and top sub-cells of a $3 \mathrm{~J}$ lattice-matched solar cell (solid dots). This figure has been modified from ${ }^{10}$.

\section{Processing results obtained with solar cell measurement.}

1. Using calibrated isotype cell components for reference, determine the photocurrent generated by the top and middle sub-cells of the solar cell used as a light sensor for every position (for a detailed discussion on how to estimate top and middle photocurrents from the signals recorded during the flash decay refer to ${ }^{11}$ ).

2. Draw a graph representing the approximated photocurrent as a function of the lens-to-receiver distance for both top and middle subcells.

3. Compare the results obtained using the ADG achromatic Fresnel lens with that of the SoG Fresnel lens.

\section{Processing results obtained with CCD camera measurement.}

1. Identify the centroid of the light in the photographs taken with the CCD camera.

NOTE: The "centroid of light" of an irradiance map distribution is the center of the area whose irradiance level is above $90 \%$ of the maximum irradiance of the map.

2. Once the spot centroid is identified, define a number of possible radiuses and, for each one, calculate the percentage of light contained within the circle with respect to the total irradiance contained in the photograph.

3. Calculate the spot radius. It is defined as the radius containing $95 \%$ of the total irradiance. NOTE: A value of $95 \%$ has been chosen in order to avoid an artificially large spot due to the noise caused by light proceeding from external sources, i.e., direct light from the xenon lamp or light from the surrounding environment.

4. Repeat processing steps from 3.4.1. to 3.4.3. for measurements with a hot and cold mirror.

5. Plot a graph representing the light spot diameter as a function of the lens-to-receiver distance with respect to the optimal position (minimum spot size) for both blue and red light (hot mirror and cold mirror measurements, respectively). 


\section{Representative Results}

The most important results obtained from previously described experimental tests are the following:

- Achromatic behavior of ADG Fresnel lens has been demonstrated using CCD camera measurements (Figure 7).

- The optical efficiency (proportional to the current measured by the MJ cell used as a light sensor) of the ADG Fresnel lens shows a large tolerance when the cell is moved from the optimum focal distance and along the focal distance axis (Figure 8).

- The size of the spot cast by the ADG lens shows a large tolerance for different temperatures (Figure 9).

The evolution of the spot diameter as a function of the lens-to-receiver distance is shown in Figure 7 for both lenses, a conventional SoG Fresnel lens and the ADG Fresnel lens. The top and middle sub-cells have been analyzed separately by means of two dichroic filters, one hot mirror filtering light with a wavelength higher than $700 \mathrm{~nm}$, and one cold mirror filtering light whose wavelength is shorter than $700 \mathrm{~nm}$. In Figure 7a, it can be seen that the minima of the two curves are displaced. This is due to chromatic aberration: since the refractive index for short wavelengths is higher, the focal point for blue light is closer to the lens. Then, the minimum spot for blue light is displaced to the left (toward the lens) and the minimum spot for red light is displaced to the right (towards infinity). Conversely, in Figure 7b, it can be observed that, for the ADG Fresnel lens, the position of the minimum spot for blue light corresponds exactly with the minimum spot for red light, proving the lens exhibits achromatic behavior.

The evolution of the normalized photocurrent generated by a MJ solar cell illuminated by a concentrating lens as a function of relative celllens distance is shown in Figure 8. The wider aspect of the curve for the ADG Fresnel lens means that, thanks to the achromatic design, it has a higher tolerance to a displacement of the lens from its optimum position along the optical axis than a conventional SoG Fresnel lens. As a consequence, ADG lenses are more tolerant to assembling errors or to any phenomenon that changes the focal distance, e.g., a temperature variation.

Finally, the variation of the light spot cast by the lens as a function of lens temperature is shown in Figure 9. The top and middle sub-cells have been analyzed separately by means of dichroic filters (hot and cold mirrors). Lenses have been put inside a thermal chamber with a transparent cover glass to control their temperature ${ }^{12}$. The graphs in Figure 9 show how the temperature variation has a lower impact on the ADG Fresnel lens than on the reference SoG Fresnel lens. In fact, for the latter, for a temperature increment of $20^{\circ} \mathrm{C}$, the expansion of the light spot size is significant: the diameter is about $30 \%$ larger for the top sub-cell and up to $60 \%$ larger for the middle sub-cell. On the contrary, for the ADG lens, even in the worst case the increase is below $20 \%$. That means that even in outdoor operating conditions with strong thermal excursion, using the ADG lens would make the system performance more stable.

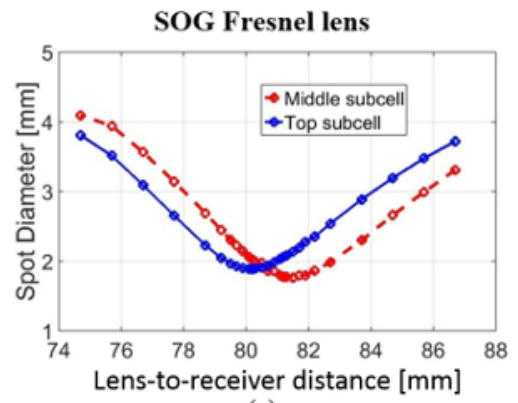

(a)

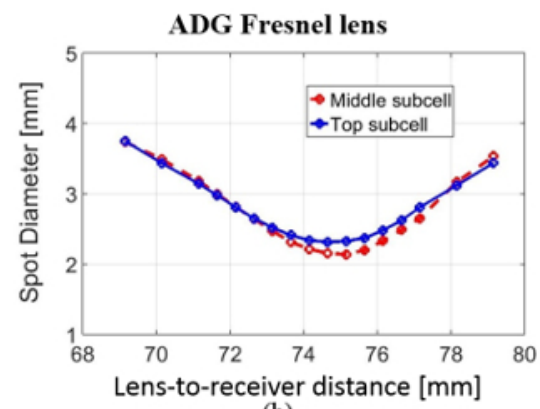

(b)

Figure 7. Measured spot diameter as a function of lens-to-receiver distance. Spot diameter is defined as that including $95 \%$ of the energy. Red dashed lines represent spot diameters for longer wavelengths (those typically converted by the middle sub-cell in MJ solar cells, i.e., $650-900$ $\mathrm{nm}$ ) and blue continuous lines represent spot diameters for shorter wavelengths (those typically covered by the top subcell, i.e., $350-650 \mathrm{~nm}$ ). (a) SOG Fresnel lens, (b) ADG Fresnel lens. This figure has been modified from ${ }^{8}$. Please click here to view a larger version of this figure. 


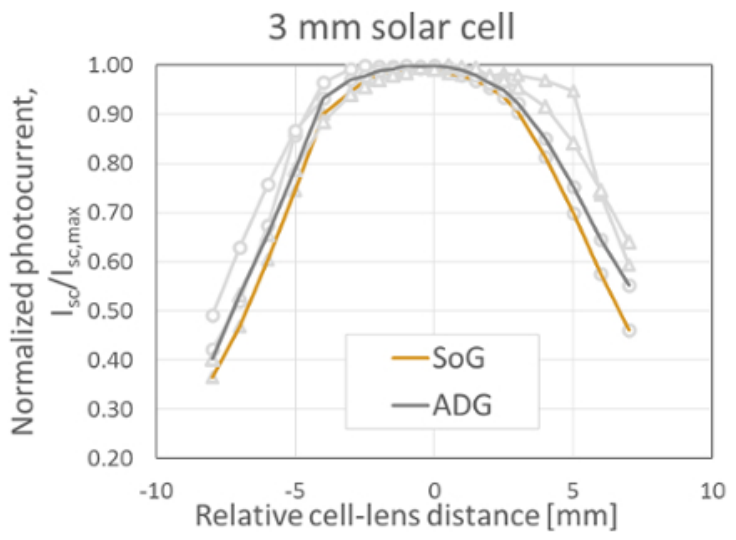

Figure 8. Normalized photocurrent generated by a MJ solar cell whose diameter is $3 \mathrm{~mm}$ as a function of the relative cell-to-lens distance. Each curve has been divided by its maximum value. The zero in the x-axis for three lenses represents the optimal focal distance (where the spot minimizes). Background curves represent the normalized photocurrents generated by the top (circular markers) and middle (triangular markers) sub-cells. ADG_v2 is an improved ADG Fresnel lens design. The normalized current produced by the MJ solar cell (the minimum value between the top and middle photocurrents) has been remarked for the sake of clarity. This figure has been modified from ${ }^{13}$. Please click here to view a larger version of this figure.
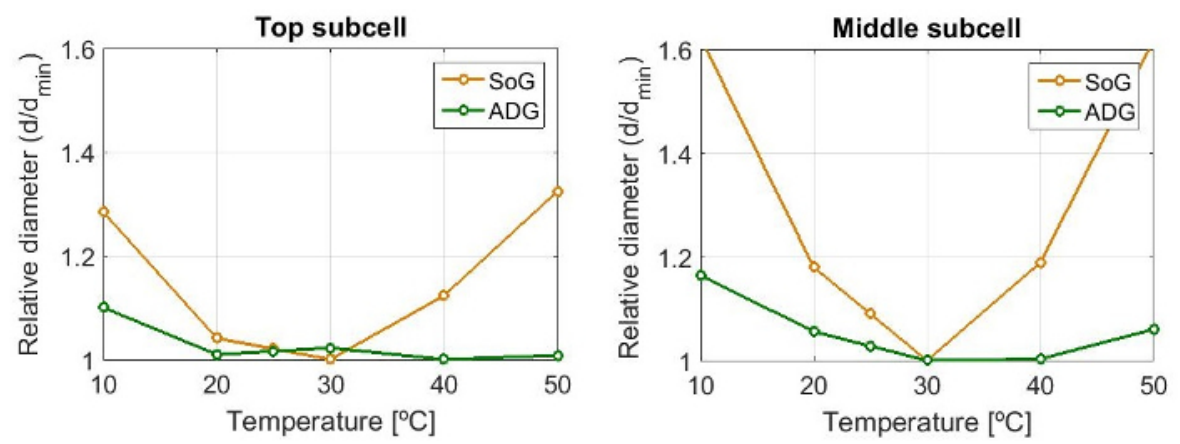

Figure 9. Relative spot size as a function of the lens temperature. (A) Results related to the top sub-cell (measurement carried out using a dichroic hot mirror filter). (B) Results related to the middle sub-cell (measurement carried out using a dichroic cold mirror filter). The relative spot size is obtained dividing the spot size by the minimum value measured for each lens. This figure has been modified from ${ }^{13}$. Please click here to view a larger version of this figure.

\section{Discussion}

The method proposed for the characterization of ADG Fresnel lenses includes two different procedures: the first one uses solar cells as light sensors, while the second is based on a CCD camera.

Applying the solar cell based procedure, the photocurrent generated by a MJ solar cell has been measured using different Fresnel lenses as concentrators. As described in the protocol, the CPV solar simulator makes use of a xenon flash lamp emitting light that is reflected on a parabolic mirror. Such a mirror generates a collimated light beam on the measuring plane (coincident with the lens aperture). Due to the mirror manufacturing tolerances and surface roughness, the collimated light is not uniform on the measuring plane. The non-uniformity of the irradiance created by the solar simulator is the main source of error in our experimental measurements ${ }^{10}$. Since large lenses integrate the irradiance at the measuring plane over a large area, the error due to non-uniformity depends on the size of the lens. The solar simulator for CPV systems used at the Solar Energy Institute attains a uniformity better than $\pm 5 \%$ for $3 \times 3 \mathrm{~cm}$ optics ${ }^{9}$. For the ADG Fresnel lens tested here, whose optical aperture is $40 \times 40 \mathrm{~mm}$, the effect of non-uniformity over the measurement can be critical. In order to reduce this uncertainty, a reference lens is re-measured prior to conducting any experiment. Moreover, when carrying out these measurements, it is paramount to be especially careful during the alignment of the cell and the lens. In fact, the solar cell has to be placed exactly centered with the light spot cast by the lens in order to avoid misalignment, because if a bad initial positioning is used, the photocurrent reduction due to defocusing is altered. Another error that may occur is that caused by different shading factors of the front metallization grid (the MJ solar cell used as a sensor is calibrated using uniform irradiance but the lenses cast a Gaussian shape profile on it during the measurements). To ensure that the metallization is not affecting experimental results, it is useful to carry out several measurements displacing the lens and, as a consequence, the light spot on the receiver plane. If the measured photocurrent varies significantly when slightly moving the light spot, it means that the metallization grid is affecting the measurements.

There are other methods suitable to measure the optical efficiency of a primary lens, e.g., using thermal irradiance sensors such as thermopiles ${ }^{10}$. The main drawback of this approach is that the response of a thermal sensor is too slow for any flash-light source. Therefore, it can only be applied to outdoor measurements (which are very sensitive to the spectral distribution of irradiance and other weather conditions). With the proposed method, this limitation is avoided. 
Additionally, using the solar cell based procedure, it would also be possible to obtain the size of the light spot cast by a lens. To do so, the photocurrents generated by several $\mathrm{MJ}$ solar cells of the same type and different but similar sizes need to be measured. For the cells whose size is smaller than the light spot cast by the lens, the measured photocurrent diminishes as the cell surface decreases due to the light spilling out of the cell. Conversely, the photocurrent remains constant for MJ solar cells whose size is larger than the light spot, since regardless of the cell surface, all the light transmitted by the lens reaches the solar cell. Therefore, the size of the light spot is equal to the size of the smallest cell that attains the maximum efficiency. For this method, the higher the number of solar cells used, the higher the resolution.

Since a set of solar cells suitable to carry out the described measurements is not always available, the CCD camera procedure has been proposed to measure the light spot size. Thanks to the wide dynamic range of the CCD sensor, using photographs of the light spot taken with the camera, an accurate comparison between peak and valley values is possible. In order to calculate the absolute value of the irradiance, a calibration of the whole set-up, including the filters and CCD camera, would be necessary. Nevertheless, from the photographs, it is possible to separate the illuminated area from the dark area over an image and, thus, estimate the light spot size. The main drawbacks of this technique are the spectral mismatch between the CCD sensor and a MJ solar cell and the noise produced by sources of light different from the collimated beam generated by the solar simulator. Regarding the first problem, by adding a hot or cold mirror to the CCD camera, it is possible to obtain a spectral response very similar to that of the top and middle sub-cells (see Figure 6). Additionally, in order to limit the background noise, it is necessary to completely darken the chamber of the CPV simulator. Since it is almost impossible to completely avoid external light sources, the image processing is very important and has to be well programmed. The most critical step is the elimination of background noise. Noise filtering can be partially automated but, due to the strong dependence with external factors that are scarcely predictable, every processed image undergoes a visual examination.

The CCD procedure can be used to obtain the evolution of the light spot size as a function of the lens temperature by adding to the system a thermal chamber where lenses are placed. In this case, besides the error sources previously described, uncertainty arises from the lens temperature measurements. The control thermocouple (the one directly connected to the computer) does not represent the real lens temperature because the sensor is placed in a point of the thermal chamber close but not directly connected to the lenses to be measured. Therefore, the temperature measured using such a thermocouple is an average temperature of the environment surrounding the lenses and it does not necessarily correspond to the real lens temperature. That is why connecting each lens to an independent thermocouple is recommended. Nevertheless, there is probably a temperature gradient between different points of the lens. In order to quantify this uncertainty, once the thermal chamber achieves the desired temperature, and before performing any measurement, it is better to wait 15-20 minutes to let the system temperature become as uniform as possible.

\section{Disclosures}

We have nothing to disclose.

\section{Acknowledgements}

This work has been partially supported by the Spanish Ministry of Economy and Competitiveness under the Acromalens project (ENE2013-45229-P) and it has received funding from the European Union's Horizon 2020 research and innovation program within the project CPV Match under grant agreement No 640873.

\section{References}

1. Green, M.A., Emery, K., Hishikawa, Y., Warta, W., and Dunlop, E.D., Solar cell efficiency tables (version 49), Prog. Photovolt. Res. Appl. 24, 3 (2016).

2. Lorenzo, E. and Sala, G., in Sun II., pp. 536-539 (1979).

3. Victoria, M. New Concepts and Techniques for the Development of High-Efficiency Concentrating Photovoltaic Modules., PhD, E.T.S.I. Telecomunicación (UPM), (2014).

4. Vallerotto, G. et al. Design and modeling of a cost-effective achromatic Fresnel lens for concentrating photovoltaics. Opt. Express. 24, A1245 (2016).

5. Victoria, M., Domínguez, C., Antón, I., Sala, G., Comparative analysis of different secondary optical elements for aspheric primary lenses, Optics Express., 17, 6487-6492 (2009).

6. Languy, F. et al. Flat Fresnel doublets made of PMMA and PC: combining low cost production and very high concentration ratio for CPV. Opt. Express. 19 Suppl 3, A280 (2011).

7. Hecht, E. Optics, Third Edition. Addison Wesley Longman, Inc. (1998).

8. Vallerotto, G., Askins, S., Victoria, M., Antón, I., and Sala, G., A novel achromatic Fresnel lens for high concentrating photovoltaic systems in AIP Conf. Proc. (AIP Publishing), 050007 (2016).

9. Domínguez, C., Antón, I., and Sala, G., Solar simulator for concentrator photovoltaic systems, Opt. Express. 16, 14894 (2008).

10. Victoria, M., Askins, S., Herrero, R., Antón, I., and Sala, G., Assessment of the optical efficiency of a primary lens to be used in a CPV system. Solar Energy. 134, 406-415 (2016).

11. Domínguez, C., Antón, I., Sala, G., and Askins, S., Current-matching estimation for multijunction cells within a CPV module by means of component cells: Current-matching estimation for MJ cells within a concentrator. Prog. Photovolt. Res. Appl. 21, 1478 (2013).

12. Askins, S., Victoria, M., Herrero, R., Domínguez, C., Antón, I., and Sala, G., Effects of Temperature on Hybrid Lens Performance, in (AIP Publishing.), pp. 57-60 (2011).

13. Vallerotto, G., Askins, S., Victoria, M., Antón, I., and Sala, G. Experimental Characterization of Achromatic Doublet on Glass (ADG) Fresnel Lenses, in AIP Conf. Proc. AIP Publishing (2017). 